\title{
Assessment of combustion noise in a premixed swirled combustor via Large-eddy simulation
}

\author{
Camilo F. Silva* and Matthieu Leyko ${ }^{\dagger}$ \\ CERFACS, 31057 Toulouse, France \\ Franck Nicoud $\ddagger$ \\ Université Montpellier II, 34095 Montpellier, France \\ Stéphane Moreau § \\ Université Sherbrooke, 2500, boul. de l'Université Sherbrooke (Québec), Canada J1K 2R1
}

\begin{abstract}
Today, much of the current effort in combustion noise is the development of efficient numerical tools to calculate the noise radiated by flames. Although unsteady CFD methods such as LES or DNS can directly provide the acoustic field radiated by noise sources, this evaluation is limited to small domains due to high computational costs. Hybrid methods have been developed to overcome this limitation. In these schemes, the noise sources are decoupled from the radiated sound. The sources are still calculated by DNS or LES codes whereas the radiated sound is evaluated by acoustic codes using an acoustic analogy.

In the present paper the assessment of combustion noise is conducted by both direct (LES) and hybrid computations in a premixed swirled combustor. Some comparisons of the sound pressure levels resulting from both approaches are shown, and the main differences between the two methods are explained.
\end{abstract}

keywords: combustion noise, acoustic analogy, direct computation, hybrid computation.

\section{Introduction}

Today Large Eddy Simulation (LES) has become an important tool for the simulation and post-processing analysis of turbulent flows. It offers the best promise in the foreseeable future for the estimation of noise from flows at Reynolds Numbers of interest in both open and closed systems. In aeroacoustics, LES plays an important role in the study of aerodynamical generated noise of numerous practical cases that range from air jets, high-lift devices or landing gears in an aircraft to the rear-view mirror of a car or the blades of a wind turbine $[1,2]$. On the contrary, thermoacoustics is less understood than aeroacoustics. This is caused by additional physical phenomena implied such as the addition of unsteady heat release to the turbulent flow. Yet, LES has been successfully applied to partially premixed and non-premixed open flames $[3,4,5]$ as well as to more complex cases such as gas turbine combustors. [6]

\footnotetext{
${ }^{*}$ Corresponding author. Ph.D. Student, CFD Team, 42, Av. Gaspard Coriolis; silva@cerfacs.fr. Tel: (33).(0)5.61.19.31.10

${ }^{\dagger}$ Ph.D. Student, CFD Team, 42, Av. Gaspard Coriolis; leyko@cerfacs.fr

¥Professor, Université Montpellier II, I3M - CNRS UMR 5149 - CC51, franck.nicoud@univ-montp2.fr

$\S$ Professor, GAUS, Mechanical Engineering Department, Université de Sherbrooke, stephane.moreau@usherbrooke.ca.
} 
Computational techniques for the estimation of sound can be classified into two broad categories: direct computations and indirect, or hybrid, computations. LES is present in both categories. Direct computations resolve the flow field together with the sound radiation. A compressible LES code is therefore required in addition to high-resolution numerical schemes in order to minimize both dispersion and dissipation. Moreover, the computational domain must be large enough to include the sources of noise as well as part of the acoustic near field [7]. Very expensive computational costs can arise since hydrodynamic and acoustic scales differ by a large amount in typical applications where the Mach number is moderate. This is even more severe when dealing with thermoacoustics since the transport equation of each species must be considered in order to solve the problem of compressible multicomponent reactive flows.

In hybrid approaches, the computation of sound is made in two different steps. The sources of noise are modeled first, which requires a proper estimation of the flow and the flame dynamic properties. These sources are assumed independent of any acoustic quantity. The far field acoustic radiation is then predicted from the different noise sources. Acoustic propagation is calculated based on equations relevant to acoustic phenomena. The derivation of a wave equation governing sound propagation in an arbitrary mean flow (and therefore accounting for mean flow-acoustic interactions) remains a difficult and controversial task in aeroacoustics [8]. Since the sources of noise and the acoustic radiation are computed separately, the computational effort is less critical than in direct sound computations. The sources of noise can be computed by numerical codes with lower-resolution schemes provided that numerical dissipation is carefully controlled [9] and that the acoustic source formulations fulfill true radiation characteristics (dipole, quadripole, etc). Regarding thermoacoustics, and more specifically combustion noise, it has been established that turbulent flames behave like low frequency monopoles [10]. After the different sources have been computed, the sound radiation, due to these sources, is evaluated by solving the wave operator coming from an acoustic analogy equation $[4,5,11]$. Notably, Strahle's formulation of Lighthill's analogy $[12,13]$ which takes into account the conservation equation of multicomponent reacting flows and Phillips' analogy [14]. Alternative methods include the Linearized Euler equations or its APE formulation [15, 16]. Acoustic analogies are satisfactory for open systems, i.e. when the acoustic fluctuations produced by the source propagate to the infinite and anechoic far-field. Moreover, in these cases, it is relatively easy to distinguish pure acoustics from hydrodynamic pressure fluctuations in the region of interest (farfield): hydrodynamic pressure fluctuations are negligible in the far field since they typically decay at least as the inverse third power of the distance to the sources [17]. Less is known about aeroacoustics in confined domains where acoustic and hydroynamic pressure fluctuations are both present. Interesting developments have been done to account for turbulence-body interaction [18, 19] . More recently, Schram used a modified Curle's analogy combined with a boundary element method (BEM) for evaluating the acoustic field produced by a non-compact turbulent source in a confined domain[20].

In the field of thermo-acoustics, it seems that no significative work has been done for evaluating the noise produced by confined flames using hybrid approaches. In reactive flows, confined systems might present an important interaction between the flame, the turbulent flow and the walls of the system. However if this interaction is not strongly present, the flame can be assumed independent of the acoustic field generated and acoustic analogies should apply. The general objective of this study is therefore to investigate whether acoustic analogies might be considered for the evaluation of noise in such confined domains.

\section{Combustion noise through Phillips' analogy}

The first attempt to include inhomogeneities of the mean flow into the acoustic wave operator is due to Phillips [21] who derived the following expression: 


$$
\begin{aligned}
\frac{d^{2} \pi}{d t^{2}}-\frac{\partial}{\partial x_{i}}\left(c^{2} \frac{\partial \pi}{\partial x_{i}}\right) & =\frac{\partial u_{i}}{\partial x_{j}} \frac{\partial u_{j}}{\partial x_{i}}+\frac{d}{d t}\left(\frac{\gamma-1}{\rho c^{2}} \dot{\omega}_{T}\right) \\
& +\frac{d}{d t}\left[\frac{\gamma-1}{\rho c^{2}}\left(\nabla \cdot(\lambda \nabla T)-\rho \sum_{k} Y_{k} c_{p, k} \mathbf{v}_{k} \cdot \nabla T+\tau: \nabla \mathbf{u}\right)\right] \\
& -\frac{\partial}{\partial x_{i}}\left(\frac{1}{\rho} \frac{\partial \tau_{i j}}{\partial x_{j}}\right)+\frac{d^{2}}{d t^{2}}(\ln r)
\end{aligned}
$$

where $\pi$ is a function of the logarithm of the pressure $\pi=(1 / \gamma) \ln \left(p / p_{\infty}\right)$. The first term in the RHS is related to the noise created by turbulence. The second term is the monopole source of noise due to the unsteady heat release induced by the flame. The third one is linked to the noise produced by molecular transport whereas the gradient of the viscous tensor appears in the fourth term. Finally, the last term is known as the non-isomolar combustion source of noise.

In order to simplify this equation, one may consider different realistic assumptions in order to evaluate the acoustics for low mach number reactive systems [22]. Therefore, it is stated that

- The pressure level of the oscillations are small compared to the local mean pressure. $p^{\prime} / p_{0} \ll 1$.

- The system is nearly isobaric so that $p_{0} \approx$ const.

- The mean flow is small so that the convective terms in the equation are negligible.

As a consequence, the acoustic wave equation for low mach number reacting flows reads

$$
\nabla \cdot\left(c_{0}^{2} \nabla p^{\prime}\right)-\frac{\partial^{2} p^{\prime}}{\partial t^{2}}=-(\gamma-1) \frac{\partial \dot{q}^{\prime}}{\partial t}-\gamma p_{0} \nabla \boldsymbol{v}: \nabla \boldsymbol{v}+\frac{\gamma p_{0}}{W_{0}} \frac{\partial^{2} W^{\prime}}{\partial t^{2}}
$$

where $c_{0}, p, \gamma, \dot{q}, \boldsymbol{v}, W$ represent the speed of sound, the pressure, heat capacity ratio, the heat release rate, the velocity vector and the mixture molar weight respectively. The symbols ()$_{0}$ and ()$^{\prime}$ define mean and fluctuating quantities respectively. As it can be noticed in the left hand side of Eq. 2, the speed of sound $c$ is placed inside the divergence operator. This ensures to capture acoustic fluctuations with strong variations of the mean temperature as it occurs close to the flame front.

In the combustion case exposed in this paper, the non-isomolar combustion noise does not play an important role since the reactant mixtures are highly diluted in nitrogen. Further on, the aerodynamic source of noise is considered small with respect to the noise source associated with the perturbation of the heat release rate [23]. The inhomogeneous wave equation then reduces to

$$
\nabla \cdot\left(c_{0}^{2} \nabla p^{\prime}\right)-\frac{\partial^{2} p^{\prime}}{\partial t^{2}}=-(\gamma-1) \frac{\partial \dot{q}^{\prime}}{\partial t}
$$

\section{Description of the acoustic Tool}

Under harmonic oscillation assumptions, the pressure fluctuation $p^{\prime}$ and the heat fluctuation $\dot{q}^{\prime}$ are expressed as follows [24]: 


$$
\begin{aligned}
p^{\prime}(\vec{x}, t) & =\Re\left(\hat{p}(\vec{x}) e^{-i \omega t}\right) \\
\dot{q}^{\prime}(\vec{x}, t) & =\Re\left(\hat{\dot{q}}(\vec{x}) e^{-i \omega t}\right)
\end{aligned}
$$

where $\omega=2 \pi f$. The quantities $\hat{p}$ and $\hat{\dot{q}}$ are complex amplitudes which depend on space only and are related by the so called Helmholtz equation :

$$
\begin{cases}\nabla \cdot c_{0}^{2} \nabla \hat{p}+\omega^{2} \hat{p}=i \omega(\gamma-1) \hat{\dot{q}} & \text { in } \Omega \\ + \text { Boundary Conditions } & \text { on } \Gamma\end{cases}
$$

When solving eq. 6 for pressure, the combustion term $\hat{\dot{q}}$ must be either known or modeled. If thermoacoustic eigen modes are sought for, $\hat{\dot{q}}$ is considered as a function of the acoustic pressure at some reference position, $\hat{\dot{q}}=\hat{\dot{q}}_{a m p}(\hat{p}, \omega)$ so that eq. 6 is an eigenvalue problem which must be solved by using dedicated numerical methods [25]. On the contrary, when dealing with combustion noise the flame is considered as an autonomous acoustic source that generates combustion noise and $\hat{\dot{q}}$ reduces to a fixed forcing term:

$$
\left\{\begin{array}{l}
\nabla \cdot c^{2} \nabla \hat{p}+\omega^{2} \hat{p}=i \omega(\gamma-1) \hat{\dot{q}}_{n o i s e}(\omega) \\
+ \text { Boundary Conditions }
\end{array}\right.
$$

For Eq. 7 the mathematical problem to solve is a linear system:

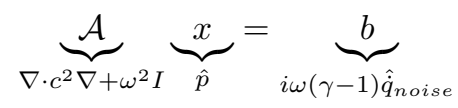

This Linear system is solved by the Generalized Minimum REsidual (GMRES) method. This algorithm was chosen mainly due to its portability, simplicity, flexibility and efficiency . GMRES solves large, sparse and non Hermitian linear systems and belongs to the class of Krylov based iterative methods. This is an important feature that allows using only Matrix-vector products when solving Eq. 8, instead of storing the full matrix $\mathcal{A}$.

The present numerical tool uses a CERFACS implementation of the GMRES algorithm for both real and complex, single and double precision arithmetics suitable for serial, shared memory and distributed memory computers [26].

\section{Experimental Configuration}

Both direct and indirect computations of combustion noise using the above methodology are assessed by comparing with the experiment in a swirled premixed combustor (EC2 combustor) carried out in the laboratory EM2C (École Centrale Paris) [27, 28, 29]. The EC2 combustor consists in two geometrically indentical stages for air-fuel injection, a premixer and a combustion chamber. Air is fed into each stage through a circular manifold in which a swirler is inserted. This swirler has a hollow cylinder with large lateral openings (see Fig. 1a), through which air is injected in the inner premixer channel. Inside these rectangular openings, four injectors $(1 \mathrm{~mm}$ diameter) deliver gaseous propane perpendicularly to the air flow. This cross-flow configuration enhances fuel-air mixing. The tangential injections create a strong 
swirl motion in the $D=30 \mathrm{~mm}$ diameter inner channel, which in turn generate a central recirculation zone at the plenum that stabilizes the flame. The flame is controlled by the fuel-air ratio imposed in each of the two stages and is considered premixed and compact. Note that the LES could consider modeling the lines at each stage of both fuel and air as shown in Fig. 1(a). Nevertheless, important computational costs would arise due to the small grid cells that would be necessary to mesh the fuel lines. Since an homogeneous air-fuel mixing is considered to be achieved before arriving to the reacting zone, the fact of meshing fuel lines far upstream from the flame might be totally unnecesary. Therefore the present LES will only consider the air lines (simplified model shown in Fig. 1b) in which already a premixed mixture with equivalent ratios $\phi_{1}-\phi_{2}$ is accounted for each stage repectively.

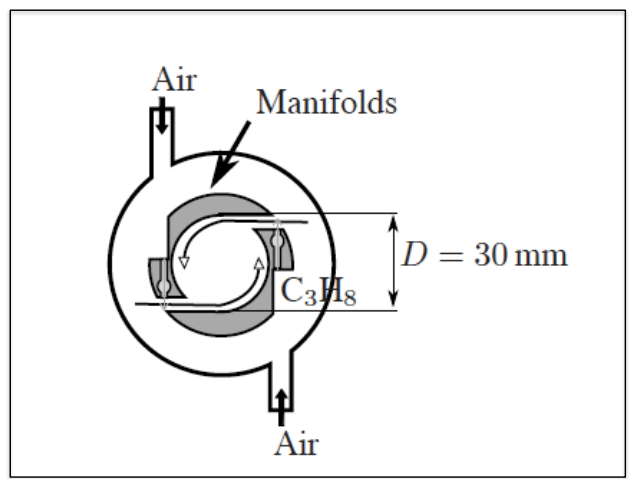

(a) Hollow cylinder - Actual test rig

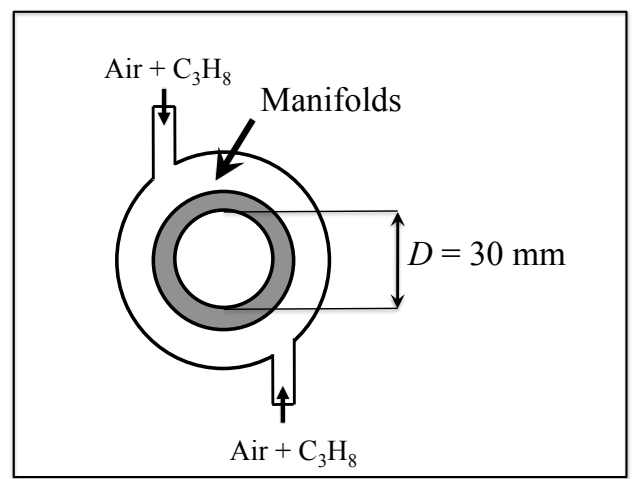

(b) Hollow cylinder simplified for LES

Figure 1: Schematic view of a tranversal section through the premixer and circular manifolds.

This configuration features strong combustion instabilities depending on the fuel staging ratio $\alpha$, defined as the ratio of the fuel massflow in the furthest stage from the chamber (stage 1) to the total fuel injected massflow.

$$
\alpha=\frac{\dot{m}_{f, 1}}{\dot{m}_{f, 1}+\dot{m}_{f, 2}}
$$

These instabilities are characterized by an important noise radiation due to the intense acoustic levels reached within the chamber and the premixer. The regime in the present study is given in Table 1. The gas mixture in the plenum is considered as perfectly premixed with a global equivalence ratio of $\phi_{g}=0.832$.

\begin{tabular}{|c|c|c|c|c|c|c|c|}
\hline$\alpha$ & $\dot{m}_{\text {air }, 1}$ & $\dot{m}_{f, 1}$ & $\dot{m}_{\text {air }, 2}$ & $\dot{m}_{f, 2}$ & $\phi_{1}$ & $\phi_{2}$ & $\phi_{g}$ \\
\hline $14.5 \%$ & 20 & 0.20 & 20 & 1.20 & 0.238 & 1.428 & 0.832 \\
\hline
\end{tabular}

Table 1: Present operating regime (Mass flow $\dot{m}$ in $m^{3} / h$ ).

The combustion chamber is made of two quartz windows for flame visualizations, and two refractory concrete plates (top and bottom) which can be equipped either with small quartz windows for Particle Image Velocimetry (PIV) laser measurements or with transducer ports for acoustic measurements. The three PIV planes we are comparing with are shown in Fig. 2. The combustion chamber and the premixer are also equipped with seven microphones (denoted M1 to M7 in Fig. 2) placed at equal distances along the combustor. 


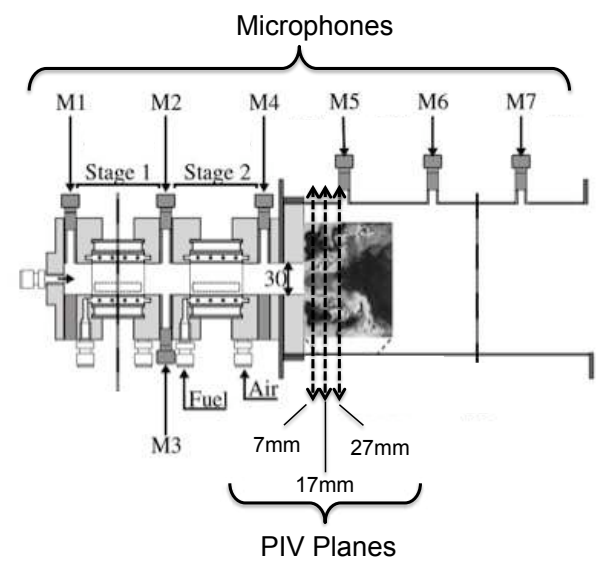

Figure 2: Two staged swirled premixed combustor. (Courtesy of École Centrale Paris)

\section{Combustion noise Analysis}

\subsection{Direct Approach}

AVBP, developed by CERFACS, is the parallel solver used for the LES computations [30]. In this code, the full compressible Navier Stokes equations are solved on hybrid (structured and unstructured) grids with second order spatial and temporal accuracy. Subgrid scale stresses are described by the Smagorinksy model. The flame/turbulence interactions are modeled by the Dynamic Thickened Flame (DTF) model [31]. This combustion model has been used in numerous studies of turbulent combustors [32, 40, 41][42] in which it has been shown to well predict ignition, blow-off and flash-back of flames as well as acoustic-flame interactions in specific configurations. The spatial discretization in AVBP is based on the finite volume method with a cell-vertex approach, combined to a numerical scheme derived from the Lax-Wendroff scheme. AVBP has been validated/used for a considerable number of configurations.[32, 33, 34]

Boundary Conditions in AVBP are treated by the Navier Stokes Characteristic Boundary Conditions (NSCBC) method [35]. This method is already an standard technique to control wave crossing the boundaries $[36,37,38]$. It consists in decomposing the variation of flow variables on boundaries into terms due to ingoing and outgoing waves. While walls are always taken as totally reflecting surfaces (no velocity fluctuations normal to the surface are allowed $\rightarrow u^{\prime}=0$ ) with a reflection coefficient $R=|R| e^{j \theta}$ $(|R|=1$ and $\theta=0)$, the same cannot be stated for inlet and oulets. Acoustics of both inlets and outlets is characterized by their acoustic impedance $Z=p^{\prime} /\left(\rho_{0} c_{0} \vec{u}^{\prime} \cdot \vec{n}\right)$. Following [38] it is possible to relate this acoustic quantity to the NSCBC parameters and is therefore straighforward to consider in the LES. This procedure was carried out with experimental data provided in [27, 28, 29]. Similar results were obtained when simplified acoustic conditions $\left(u^{\prime}=0\right)$ were applied, proving that acoustic conditions at the inlets are not critical in the present LES study. Classically, outlets to the atmosphere are considered restrictive to pressure fluctuations $\rightarrow p^{\prime}=0(|R|=1$ and $\theta=\pi)$. It is though preferred to extend the computational domain to some region of the atmosphere after the burner outlet. By doing so, the true impedance of the outlet is implicitely accounted for.

Two different meshes were used to compute the flow and flame dynamics of the EC2 combustor. The 'coarse' mesh has 3 millions of cells whereas the 'refined' mesh is made of 10 million cells. The quality of the two LES performed is evaluated through Pope's criterion [39]. The smagorinsky filter and the grid resolution for both cases should be sufficient to resolve at least $80 \%$ of the energy remote from the 
wall. The ratio of the resolved turbulent kinetic energy $k_{f}$ with respect to the modeled turbulent kinetic energy $k_{s g s}$ is then quantified.

$$
Q_{L E S}=\frac{k_{f}}{k_{f}+k_{s g s}}
$$

where $k_{f}=\frac{1}{2} \tilde{u}_{i} \tilde{u}_{j}$ and $k_{s g s}=\frac{1}{2} \widetilde{u_{i} u_{j}}-\frac{1}{2} \tilde{u}_{i} \tilde{u}_{j}=\frac{3}{2}\left(u_{s g s}^{\prime}\right)^{2}$ and $\widetilde{()}$ stands for the LES filter. Figure 3 demonstrates that, for both LES cases, $Q_{L E S}$ is greater than 0.8 for almost the entire computational domain excepting regions near walls. The premixer turbulence is however better captured by the 'refined' mesh. It is well known that extremely high computational costs arise when a proper LES on boundary layers is sought. Nevertheless, boundary layers are assumed to little contribute to noise radiaton/scattering of turbulent flames. A high resolution in regions near walls is therefore not considered.

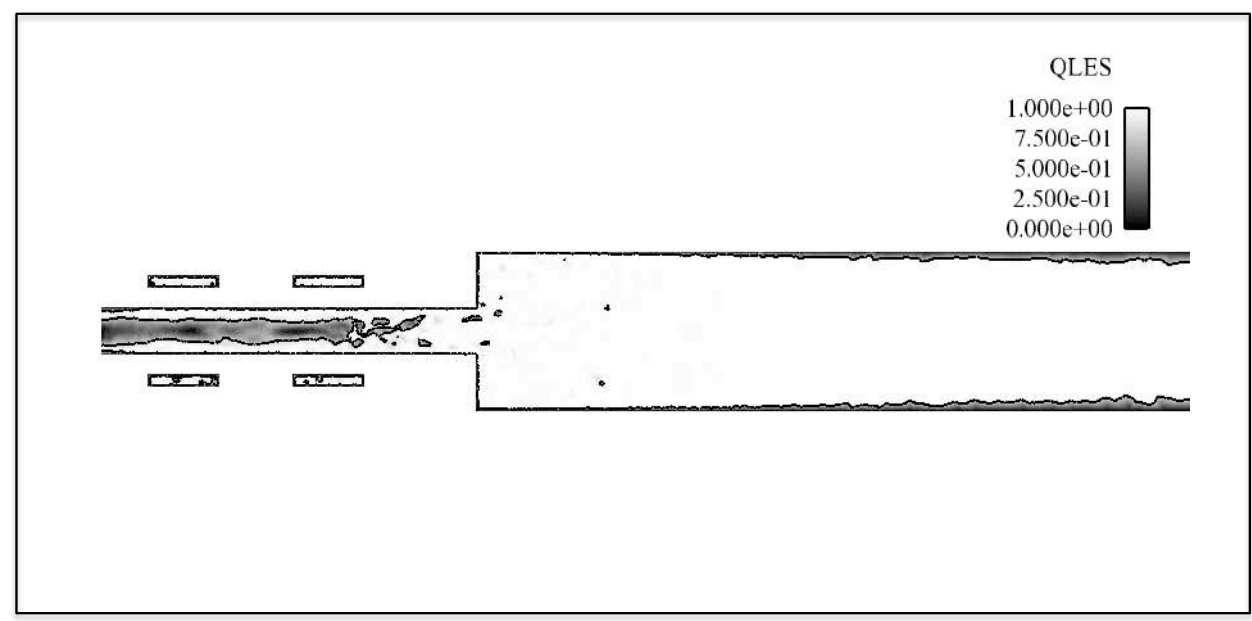

a) LES 3 million Cells

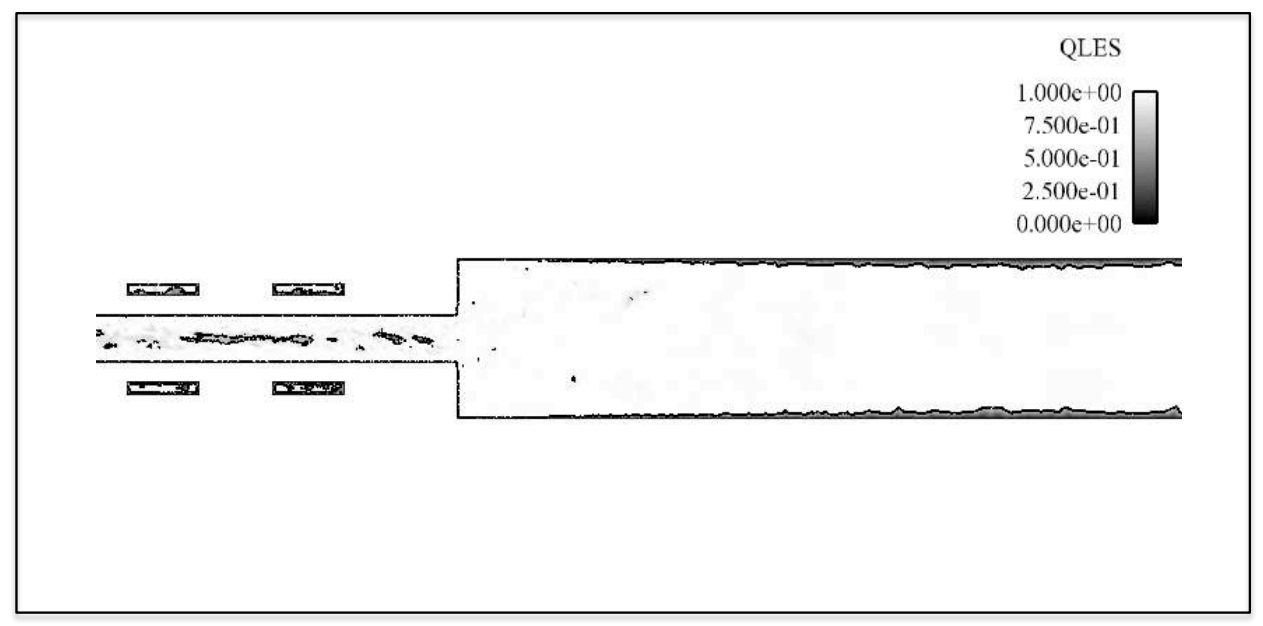

b) LES 10 million Cells

Figure 3: Instantaneous Field of Pope's Criterion. The black line stands for the isocontour line $Q_{L E S}=$ 0.8

Both meshes are found to reproduce the mean PIV very well. This can be observed in Fig. 4. Both 
LES succeed in predicting the central recirculation zone satisfactorily. The LES on the 'refined' mesh is however more accurate for the outer region, particularly for the radial velocity. The fluctuating velocity field is characterized by rms profiles. Figure 5 shows that on the 'coarse' mesh a high overprediction of velocity fluctuations is obtained in both axial and radial components. On the fine grid however the LES clearly recovers the experimental velocity fluctuating field.

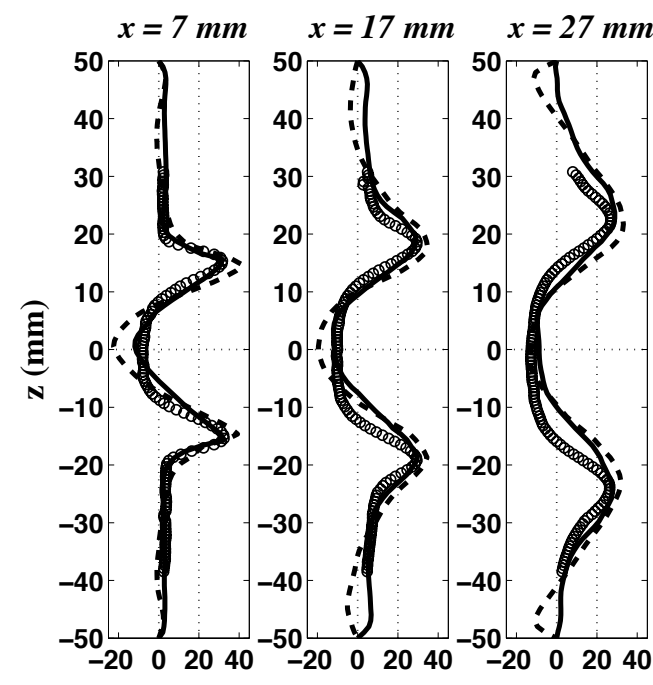

(a) Mean Axial Velocity $(\mathrm{m} / \mathrm{s})$

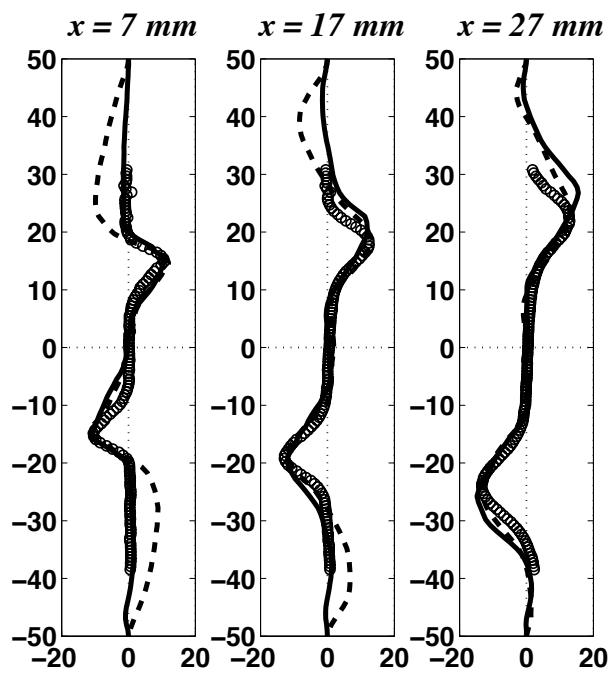

(b) Mean Radial Velocity $(\mathrm{m} / \mathrm{s})$

Figure 4: Velocity Profiles: ○ Experimental PIV measurements - - - LES 3 million cells, — LES 10 million cells

Acoustics and flame dynamics of the system represented by the heat release are, on the contrary, more difficult to evaluate than the mean and fluctuating velocity field. The mean value of heat release is similar in both LES and is close to the 40kW experimental thermal power, as can be observed in Fig. 6(a). Different values in the variations of heat release are however obtained for each LES. Strong and more regular fluctuations of heat release are obtained with the 'coarse' mesh while smaller and less periodic fluctuations are given by the 'refined' mesh computation. It is likely that the coarser mesh does not capture enough small turbulent scales and trigger too large turbulent eddies. These large coherent structures might clearly have an influence on the flame dynamics and thus in the large fluctuations of heat release. The value of the rate of change of heat release integrated over the whole volume of the combustor has also been computed for the two different meshes and is shown in Fig. 6(b). On the finer mesh a quieter flame is modeled, considering the smaller values of rate of change of heat release compared to those obtained from the coarse mesh. As a consequence, smaller rms pressure values should be expected on the finer mesh. Acoustics in the chamber is rather characterized by the Sound Pressure Level (SPL) at a given point than rms values of the pressure. Figure 7 compares the SPL values at the microphone 7 (see the location of $M 7$ in Fig. 2) of the computations on the refined and coarse meshes to the experimental measurements. Both LES clearly overestimate the sound levels with a significant improve on the finer. It is then found that in order to correctly evaluate the dynamics of a flame and the acoustics generated by this one it is not enough to satisfactorily model the fluctuating velocity field as shown in Figs. 4 and 5. As stated before, computing acoustic pressure fluctuations is very challenging since these values are very small compared to the aerodynamic fields. Several additional phenomena can play an important role.

First, the performed LES assume a perfect premixed mixture of air and fuel in the reactive region. This 


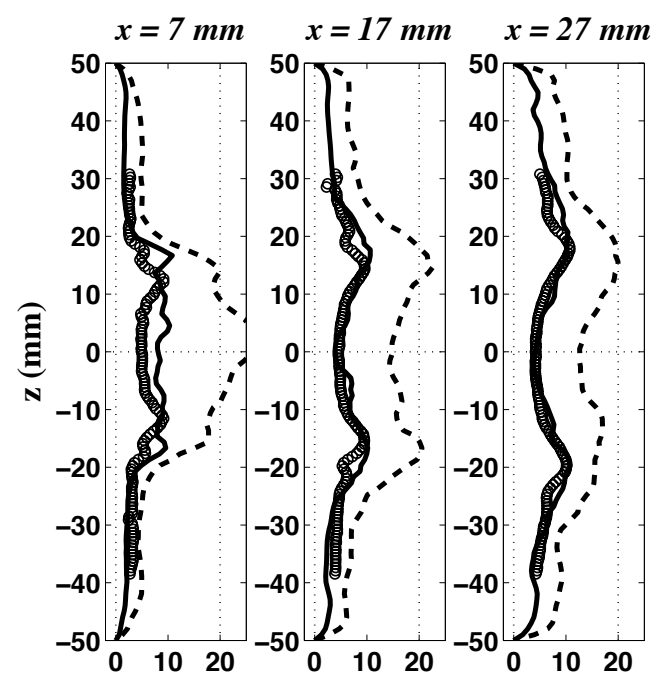

(a) RMS Axial Velocity $(\mathrm{m} / \mathrm{s})$

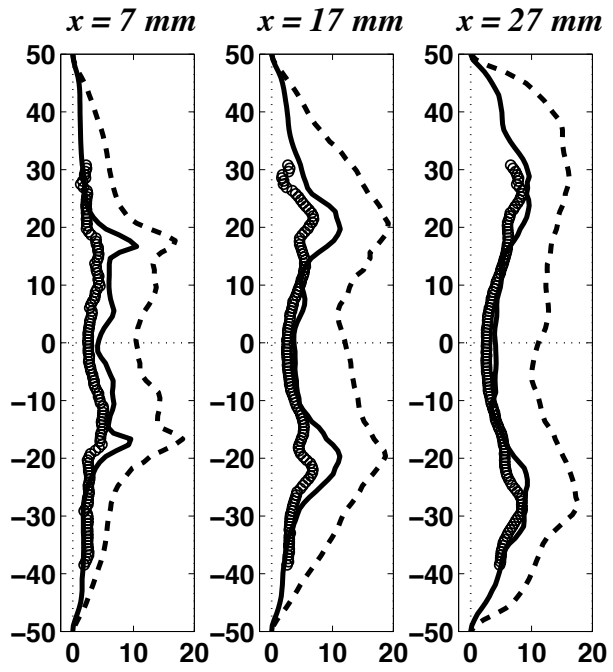

(b) RMS Radial Velocity $(\mathrm{m} / \mathrm{s})$

Figure 5: Velocity Profiles: ○ Experimental PIV measurements

- - - LES 3 million cells, — LES 10 million cells

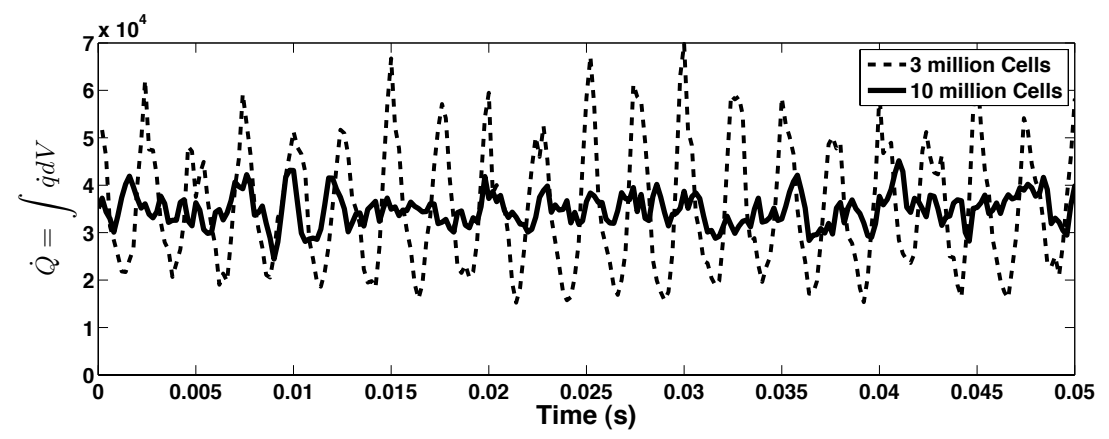

a) Heat release integrated over the entire volume $(W)$

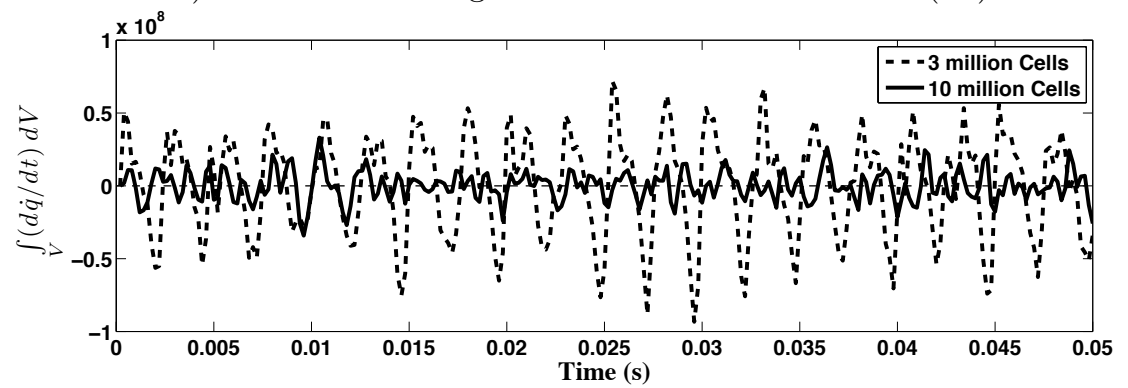

b) Rate of change of heat release integrated over the entire volume $(\mathrm{W} / \mathrm{s})$

Figure 6: Heat Release and rate of change of heat release 


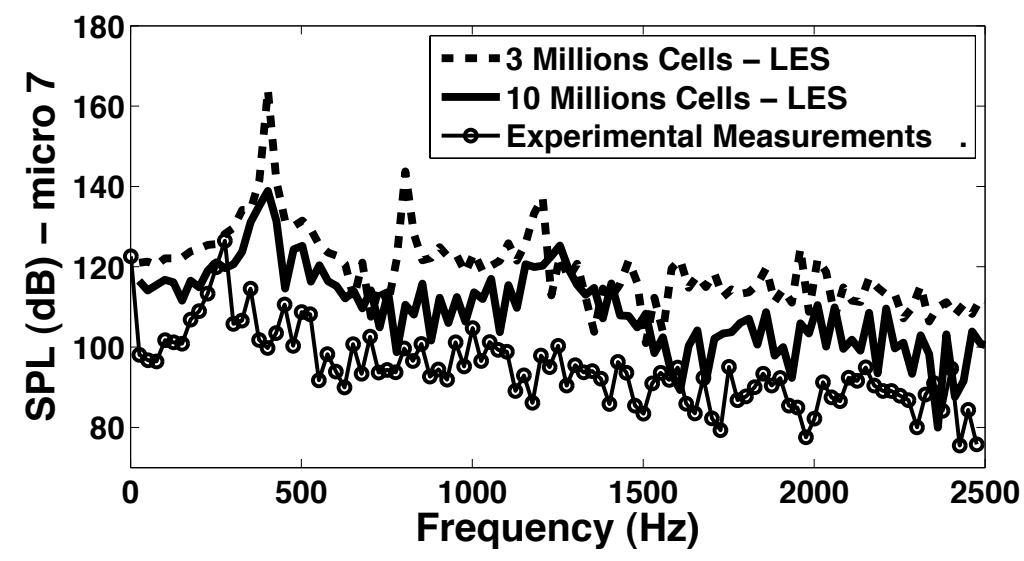

Figure 7: Sound Pressure Level

assumption might be a misinterpretation of reality. Small pockets of propane-air presenting an equivalent ratio different for $\phi_{g}$ may reach the flame influencing its dynamics and as a consequence the radiated sound. Secondly, perfect adiabatic walls were considered in the present LES. In [42] it was shown that accounting for heat transfer leads to a reduction in the SPL. Some computations, not shown here, were performed considering heat transfer by modeling approximate heat loss coefficients (as done in [42]). No significative changes on the radiated noise were observed. Nevertheless, heat transfer might still be modeled too aproximatively knowing that exact values of heat transfer coefficients are extremely difficult to be obtained experimentally. A proper simulation of conjugate heat transfer including convection, conduction and radiation might be important at some level for noise modeling in turbulent flames, but presently remains too cumbersome. Thirdly, resolution of the computation (mesh refinement and order of the numerical scheme) is clearly significant. High grid resolutions in the reactive region not only means a smaller influence of the combustion model but also to be able to account for the smallest turbulent structures present in the shear layer that might influence the coherence of the bigger scales and thus the global turbulent interaction with the flame. At last but not the least, another explanation lies in the experimental data. Measuring acoustics in a confined combustion chamber is only reliable when acoustic leakages are proved to be controlled. This is, anyway, a difficult task. It has been found by the concerned EC2 experimentalists that acoustic leakages are still not totally well managed. As a result, wrong evaluations of pressure fluctuations may arise.

\subsection{Hybrid approach}

The acoustic outputs from both direct and hybrid approaches are compared for the 10 million cells mesh independently of experimental data. As sketched in Fig. 8, hydrodynamic pressure fluctuations are assumed to be small when considering results from the direct approach. Therefore, the acoustic field resulting from the hybrid approach is directly compared to the pressure fluctuation field coming from direct computations.

The hybrid computation accounts for two steps. First, the source of combustion noise is computed by postprocessing the data obtained from the LES computation (instantaneous heat release rate in addition to mean flow parameters taken from the 10 million cells numerical results). Secondly, the simplified Phillips equation written in the zero Mach number limit given by Eq. 7 is solved in the frequency domain. 


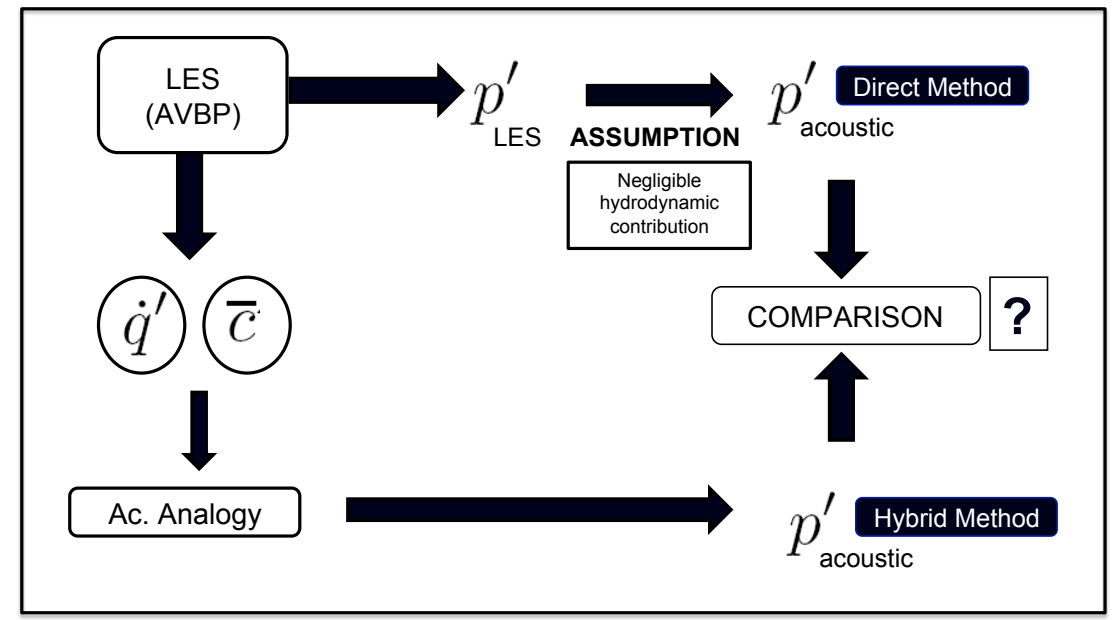

Figure 8: Exercise of comparison: Direct Approach Vs Hybrid Approach

Overall good agreement is found between both direct and hybrid approaches in Fig. 9 which shows the sound pressure levels obtained by microphones 5 and 7 (see the location of $M 5, M 7$ in Fig. 1).

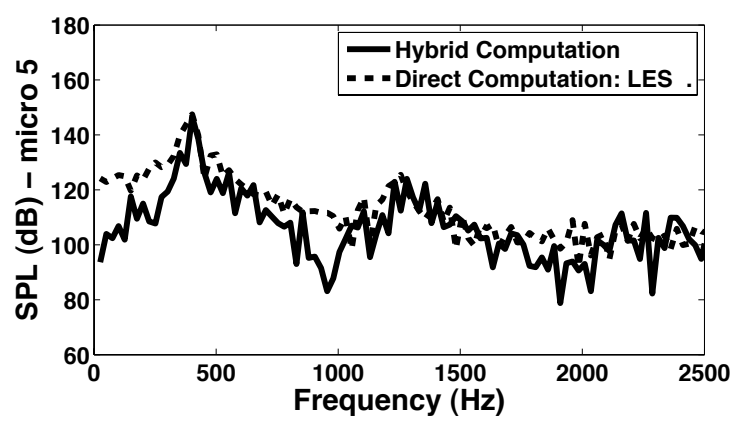

(a) Microphone 5

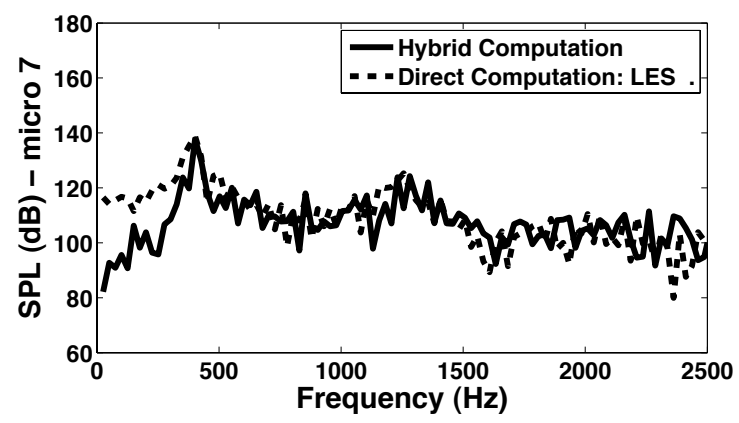

(b) Microphone 7

Figure 9: Sound Pressure Levels from the direct and hybrid approaches

It is interesting to notice that the hybrid computation manages to recover not only the magnitude of the acoustic pressure over almost all the spectrum, but also the shape of the acoustic waves. Figure 10 shows the strongest acoustic wave, the quarter wave mode, that resonates at $377 \mathrm{~Hz}$. The pressure fluctuations along the axis of the combustor at different times within a cycle are observed. As both methods yield the same envelope of variations at this frequency, the pressure fluctuation recovered by the direct computation can be seen as almost completely caused by acoustics.

In Fig. 9 some zones of the spectrum still show important gaps between hybrid and direct computations. For example in Fig. 11 two different types of pressure waves are observed at microphone 5 for the direct and hybrid computations in the region around $1000 \mathrm{~Hz}$. Whereas a pure acoustic standing wave is obtained by the hybrid approach, a perturbed pressure wave is obtained in the direct computation results. A pure standing acoustic wave can naturally have an acoustic pressure node. If this pressure node is present close to the region of the measurement device a low value of pressure fluctuation will be obtained. This is what happens for microphone 5 in the zone close to $1000 \mathrm{~Hz}$ (fig. 11a). Obviously, when the pressure fluctuations not only contain acoustics but also hydrodynamic perturbations as in the 
direct computations (Fig. 11b), no pressure node can be observed and the resulting SPL is much higher than in the hybrid computation case. Similar conclusions can be drawn at very low frequencies (before the peak at $377 \mathrm{~Hz}$ ) where the significant hydrodynamic contribution in the direct computation triggers higher pressure fluctuations.

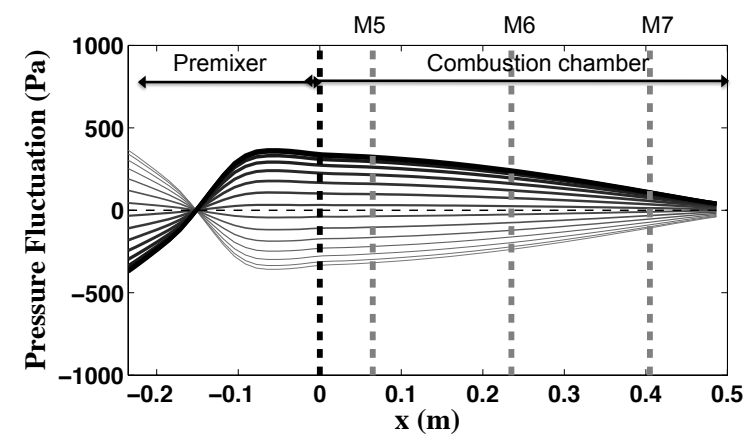

(a) Quarter pressure Wave from eq. 8 Hybrid Computation

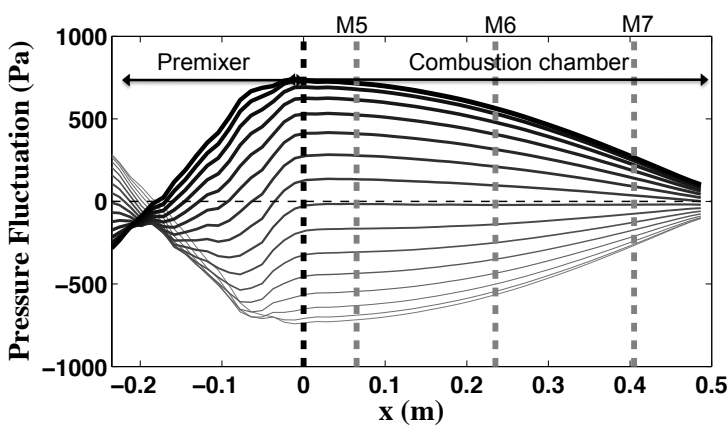

(b) Quarter pressure Wave from LES Direct Computation

Figure 10: Longitudinal pressure Waves oscillating at $377 \mathrm{~Hz}$

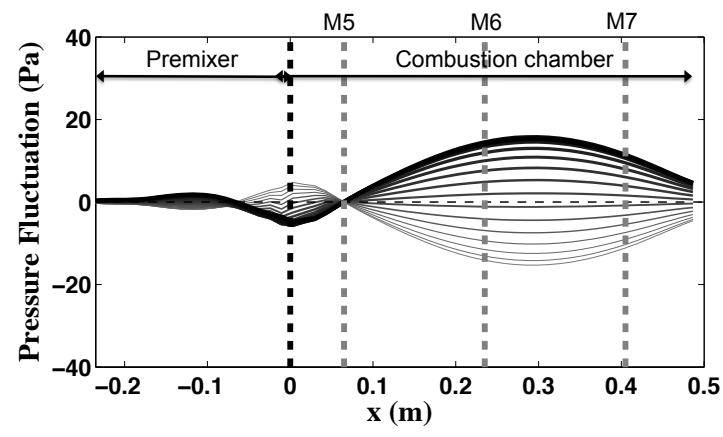

(a) Pressure Wave from eq. 8 Hybrid Computation

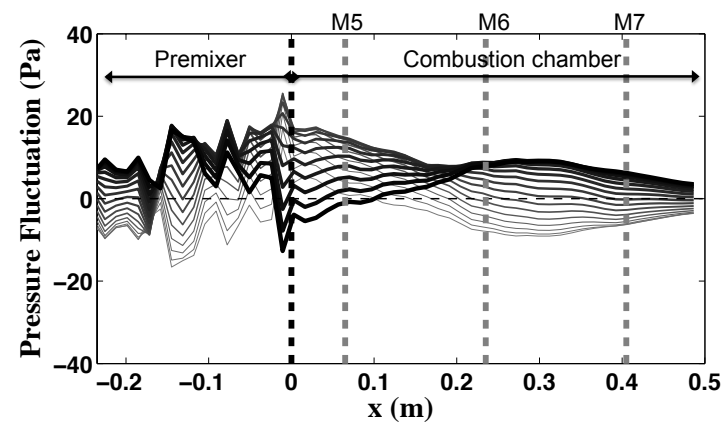

(b) Pressure fluctuation from LES Direct Computation

Figure 11: Longitudinal pressure Waves oscillating at $954 \mathrm{~Hz}$

\section{Conclusions}

Combustion noise of a premixed swirled combustor has been assessed by two different numerical approaches: a direct computation in which the noise produced by the flame is calculated together with the flow and flame dynamics, and a hybrid computation in which the acoustic field is evaluated from the sources of noise in a separate step.

Classical comparisons between mean and fluctuating ( $\mathrm{rms}$ ) velocity fields were performed between two LES on a 'coarse' mesh grid (3 million cells) and a 'refined' mesh grid (10 million cells), and PIV measurements. Mean velocity fields (axial and radial) were well predicted by both LES cases, whereas only the 'refined' mesh succeed in recovering the proper rms velocity fields. It was then observed that satisfactorily predicting the velocity fluctuating field does not mean to reproduce correct flame dynamics and heat release. On the one hand, the mean heat release corresponding to the experimental thermal 
power is well captured by both LES. On the other hand, significative differences are found between the two simulations on the shape of the instantaneous heat release and its rate of change. As a consequence, a right estimate of the combustion noise radiation is not yet well obtained. Several phenomena might be the cause of such a misprediction. A lack in numerical resolution can be one possible explanation: computing the small turbulent length scales in the shear flow region might be significant since these eddies might have a non-negligible influence on the flame dynamics and, as a consequence, on the noise prediction simulations. A higher grid resolution in the flame region also decreases the influence of the combustion model, which might have a certain effect when computing the values of rate of change of heat release. Moreover, an accurate prediction of heat transfer might also be a crucial factor, and exact modeling of convection, conduction and radiation might be important in combustion noise modeling.

The output from the hybrid computation is a pure acoustic field due to the turbulent flame. Good agreement is found in almost the entire SPL spectrum when comparing the results of both direct and hybrid computations. Nevertheless, there are still some differences in specific zones of the spectrum. Hybrid computation results only consider pure acoustic waves, and at given frequencies these pure acoustic waves may present acoustic nodes that may take place close to the acoustic sensor position. This leads to a low fluctuation of pressure at this position and hence, to a low value of the SPL spectrum at these frequencies. At low frequencies, the pressure fluctuations coming from the direct approach are most likely caused not only by acoustics but also by hydrodynamic fluctuations which then leads to higher sound pressure levels than the hybrid approach.

\section{Acknowledgments}

This work was supported by the Fondation de Recherche pour l'aéronautique et l'espace (FRAE) through the BRUCO project. The authors also gratefully acknowledge the Centre Informatique National de l'Enseignement Supérior (CINES) for giving access to parallel computers and École Centrale Paris for the interesting discussions and experimental data. An special recognition is also given to Ammar Lamraoui of the laboratory EM2C ( École Centrale Paris) for the efforts done in measuring the acoustic impedance of all fuel-air inlets on the EC2 combustion chamber.

\section{References}

[1] A. Oberai, F. Roknaldin and T. Hughes "Computation of trailing-edge noise due to turbulent flow over an airfoil" AIAA Journal, 402206 - 2216 (2002).

[2] D.J. Bodony and S. K. Lele "On the current status of jet noise predictions using Large-Eddy Simulation" AIAA Journal, 46364 - 380 (2008).

[3] H. Pitsch and H. Steiner, "Large-Eddy Simulation of a turbulent piloted methane/air diffusion flame (Sandia flame D)" Phys. Fluids , 12, 2541-2554 (2000).

[4] F. Flemming, A. Sadiki and J. Janicka "Investigation of combustion noise using a LES/CAA hybrid approach" Proc. of the Combustion Institute, 31, 3189-3196 (2007).

[5] M. Ihme, H. Pitsch and H. Bodony "Radiation of noise in turbulent flames" Proc. of the Combustion Institute, 32, 1545-1554 (2009).

[6] F. di Mare, W. Jones and K. Menzies "Large-Eddy Simulation of a model gas turbine combustor" Combust. Flame, 137, 278-294 (2004). 
[7] A. S. Lyrintzis "Integral acoustic methods: From the (cfd) near-field to the (acoustic) far-field" Int. J. Aeroacoustics, 2, 95-128 (2003).

[8] C. Bailly, C. Bogey and S. Candel "Modelling of sound generation by turbulent reacting flows" Int. J. Aeroacoustics 9, 461-489 (2009).

[9] M. Wang, S. Moreau G. Iaccarisno and M. Roger "LES prediction of wall-pressure fluctuations and noise of a low-speed airfoil" Int. J. Aeroacoustics, 3 (2009).

[10] S. Bragg "Combustion noise" J. Inst. of Fuel, 36 12-16 (1963).

[11] M. Staufer, A. Schwarz and J. Janicka "On the simulation of premixed flames and coupling of Large-Eddy Simulation with computational aeroacoustics" Acta Acustica united with Acustica, 95 409-417 (2009).

[12] M. J. Lighthill "On sound generated aerodynamically: I. General theory" Proc. R. Soc. London Ser. A, 2221 - 32 (1954).

[13] W. C. Strahle "On Combustion Generated Noise" J. Fluid Mech., 49399 - 414 (1971).

[14] S. Kotake "On combustion noise related to chemical reactions" J. Sound Vibration, 42 399-410 (1975).

[15] R. Ewert and W. Schröder. "Acoustic perturbation equations based on flow decomposition via source filtering" J. Comput. Phys., 188365 - 398 (2003).

[16] C. Bailly and D. Juvand "Numerical solution of acoustic propagation problems using Linearized Euler Equations" AIAA Journal, 3822 - 29 (2000).

[17] M. Roger "Aeroacoustics of wall-bounded flows" Von Karman Institute For Fluid Dynamics. Lecture Series. March 9-13 (2009).

[18] N. Curle "The influence of solid boundaries upon aerodynamic sound" Proc. R. Soc. London Ser. A 231, (1955).

[19] H.G. Davies, J.E. Ffowcs Williams "Aerodynamic sound generation in a pipe" J. Sound Vibration, 1612 (1981).

[20] C. Schram "A boundary element extension of Curle's analogy for non-compact geometries at lowMach numbers" J. Sound Vibration, 322 264-281 (2009).

[21] O. M. Phillips "On the generation of sound by supersonic turbulent shear layers" J. Fluid Mech., 9 $1-28(1960)$.

[22] F. Williams "Combustion theory" The Benjamin/Cummings Publishing Company (1985)

[23] H. Hassan "Scaling of combustion-generated noise" J. Fluid Mech., 49 445-453 (1974).

[24] A. Pierce "Acoustics. An introduction to its physical principles and applications" The Acoustical Society of America. (1994).

[25] F. Nicoud, L. Benoit and C. Sensiau "Acoustic modes in combustors with complex impedances and multidimensional active flames" AIAA Journal, 45 426-441 (2007).

[26] V. Frayssé, L. Giraud, S. Gratton and J. Langou "A Set of GMRES routines fo real and complex arithmetics on high performance computers." CERFACS Technical Report, (2003). 
[27] N. Tran, S. Ducruix and T. Schuller "Damping combustion instabilities with perforates at the premixer inlet of a swirled burner" Proc Combust Inst., 32 2917-24 (2009).

[28] N. Tran, S. Ducruix and T. Schuller "Passive control of the inlet acoustic boundary condition of a swirled burner at high amplitude combustion instabilities" J Eng Gas Turb Power, 131:051502 (2009).

[29] A. Lamraoui, F. Richecoeur, T. Schuller and S. Ducruix "Methodology for on the fly acoustic characterization of the feeding lines impedances in a turbulent swirled combustor" J Eng Gas Turb Power, 133:011504 (2011).

[30] AVBP Code: http://www.cerfacs.fr/4-26334-The-AVBP-code.php and http://www.cerfacs.fr/425719-Publications.php, (2010)

[31] O. Colin, F. Ducros, D. Veynante and T. Poinsot "A thickened flame model for large eddy simulations of turbulent premixed combustion" Phys. Fluids 12, 1843-1863 (2000)

[32] L. Selle, G. Lartigue, T. Poinsot, R. Koch, K.-U. Schildmacher, W. Krebs, B. Prade, P. Kaufmann and D. Veynante "Compressible Large-Eddy Simulation of turbulent combustion in complex geometry on unstructured meshes" Combust. Flame. 137, 4, 489-505 (2004)

[33] S. Roux, G. Lartigue, T. Poinsot, U. Meier and C. Bérat "Studies of mean and unsteady flow in a swirled combustor using experiments, acoustic analysis and Large-Eddy Simulations" Combust. Flame. 141, 40-54 (2005)

[34] S. Mendez and F. Nicoud "Large eddy simulation of a bi-periodic turbulent flow with effusion" J. Fluid Mech., 46, 2623-2633 (2008)

[35] T. Poinsot and S. Lele "Boundary Conditions for Direct Simulation of Compressible Viscous Flows" J. Comput. Phys. , 101 104-129 (1992).

[36] T. Poinsot and D. Veynante "Theoretical and Numerical Combustion" R.T. Edwards. (2005)

[37] N. Lamarque, M. Porta, F. Nicoud, and T. Poinsot. "On the stability and dissipation of wall boundary conditions for compressible flows" International Journal for Numerical Methods in Fluids, 62 1134-1154 (2010).

[38] L. Selle, F. Nicoud, and T. Poinsot "The actual impedance of non-reflecting boundary conditions: implications for the computation of resonators" AIAA Journal, 42 958-964 (2004).

[39] S. Pope "Turbulent Flows" Cambridge University Press. Cambridge, USA (2000)

[40] C. Martin, L. Benoit, Y. Sommerer, F. Nicoud and T. Poinsot "LES and acoustic analysis of combustion instability in a staged turbulent swirled combustor" AIAA Journal, 44 741-750 (2006).

[41] Y. Sommerer, D. Galley, T. Poinsot, S. Ducruix, F. Lacas, and D. Veynante "Large eddy simulation and experimental study of flashback and blow-off in a lean partially premixed swirled burner" Journal of Turbulence, 5 -37 (2004).

[42] A.X. Sengissen, J.F. Kampen, R.A. Huls, G.G.M. Stoffels, J.B.W. Kok and T.J. Poinsot "LES and experimental studies of cold and reacting flow in a swirled partially premixed burner with and without fuel modulation" Combust. Flame. 150 40-53 (2007) 


\section{List of Figures}

1 Schematic view of a tranversal section through the premixer and circular manifolds. . . . 5

2 Two staged swirled premixed combustor. (Courtesy of École Centrale Paris) . . . . . . . . 6

3 Instantaneous Field of Pope's Criterion. The black line stands for the isocontour line

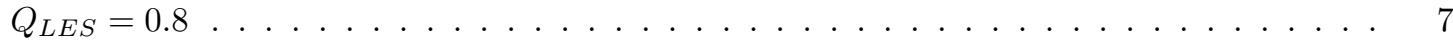

4 Velocity Profiles: $\circ$ Experimental PIV measurements . . . . . . . . . . . . . . . 8

$5 \quad$ Velocity Profiles: o Experimental PIV measurements . . . . . . . . . . . . . . . . . . 9

6 Heat Release and rate of change of heat release . . . . . . . . . . . . . . . . . . . 9

$7 \quad$ Sound Pressure Level . . . . . . . . . . . . . . . . . . . . . . . . . 10

8 Exercise of comparison: Direct Approach Vs Hybrid Approach . . . . . . . . . . . . . 11

9 Sound Pressure Levels from the direct and hybrid approaches . . . . . . . . . . . . . . 11

10 Longitudinal pressure Waves oscillating at $377 \mathrm{~Hz} \ldots \ldots \ldots \ldots \ldots$. . . . . . 12

11 Longitudinal pressure Waves oscillating at $954 \mathrm{~Hz} \ldots \ldots \ldots \ldots \ldots$ 